\title{
PARASITISME DES HÉRISSONS PAR LES MYCOTORULÉES
}

\author{
Par R.-V. TALICE
}

La mycologie parasitaire comparée est d'une très grande importance pour la connaissance plus parfaite de la mycologie humaine, dont nous ignorons encore bien des questions fondamentales. Une ćtude approfondie des blastomycoses spontanées des animaux serait très intéressante. En effet, elle pourrait apporter quelques précisions en ce qui concerne, d'une part, la hiologie des champignons parasites des cavités gastro-intestinales et respiratoires de l'homme et, d'autre part, la pathologie générale des maladies que ces champignons semblent quelquefois capables dé provoquer.

Pour ces raisons, nous croyons utile de décrire ici quelques observations que nous avons faites chez plusieurs hérissons spontanément parasités par des mycotorulées. Nous devons ce matériel à l'amabilité du Prof. Brumpt. Le fait du parasitisme d'un champignon du groupe des mycotorulées devait attirer notre attention à l'époque où nous poursuivions, avec le Dr Langeron, nos recherches sur la morphologie générale de ces parasites.

Mycotorulées chez les animaux. Historique. - Plusieurs auteurs ont.signalé des cas de parasitisme par les mycotorulées chez les animaux. Ainsi, Neumann a décrit des cas de muguet chez la poule, Delafond chez l'agneau et, d'après Blanchard, le muguet est une maladie connue aussi chez le chat, le chien, le dindon. Thiry (1913) a publié un cas de muguet spontané, sous la forme de langue pileuse brune, chez un singe adulte (Cercopithecus patas var. ruber) affaibli par une grave infection associce. Dans ce cas, le champignon a été aussi trourć à l'autopsie dans l'œsophage et l'intestin. Catanei (1925) a constaté également que le singe d'Algérie (Macacus inuus) peut héberger sur la langue un Monilia dont le développement ne s'accompagne d'aucune lésion apparente de l'organe. L'auteur a trouvé six singes porteurs du parasite sur douze examinés. D'après Brumpt, le muguet spontané s'observe aussi chez le veau et le poulain.

Malheureusement, ces auteurs n'ont pas complété leurs intéressantes observations par une description morphologique détaillée du parasite.

Axwales de Parasitologie, t. $\mathrm{X}, \mathrm{N}^{\bullet} 1 .-1^{\mathrm{cr}}$ janviev 19:32, p. 81-84.

6 . 
Observations personnelles. - Le premier cas que nous avons étudié a été constaté par le Prof. Brumpt qui, examinant les selles d'un hérisson (424) porteur de différents parasites, trouva de nombreux filaments et blastospores. Cet animal a été sacrifié quelques jours après.

Après autopsie, nous avons trouvé, par examen direct et par cultures, les champignons localisés dans l'œsophage, l'estomac, tout l'intestin, du duodénum au rectum, et finalement la trachée. Dans aucun de ces organes, nous n'avons pu constater de lésions muqueuses, macroscopiquement appréciables. L'examen soigneux de la cavité buccale ne nous a pas montré, non plus, de lésions du type du muguet. En outre, l'examen direct et la culture des sécrétions des muqueuses de l'appareil digestif ont donné des résultats négatifs. Après fixation et inclusion, nous avons aussi fait des coupes histologiques de différents segments de l'intestin grêle et du gros intestin, que nous avons colorées par l'hémalun-éosine. L'examen de ces coupes ne nous a pas montré de lésions histologiques de la muqueuse; dans quelques endroits, nous avons pu voir des blastospores bourgeonnantes englobées dans le mucus et placées soit à Ia surface interne de la muqueuse, soit dans l'intérieur des cavités glandulaires.

Les blastospores étaient rares dans l'estomac, rares dans le duodénum et de plus en plus abondantes au fur et à mesure qu'on progressait dans le tube intestinal de l'animal. Le contenu fécal du rectum montrait, à l'examen microscopique, une véritable culture, constituée par des blastospores plus ou moins bourgeonnantes et par des filaments mycéliens vrais. La réaction des selles était franchement acide au papier de tournesol.

Plus tard, nous avons autopsié d'autres hérissons et poursuivi nos recherches en employant la même technique : ouverture de tout le tube gastro-intestinal ; prélèvement du mucus de l'estomac, de l'intestin grêle et du rectum ; examen d'une préparation colorée par le liquide de Lugol fort; culture en liquide de Raulin acide. Ce dernier milieu, dès le premier ensemencement, exceptionnellement après un premier ou un deuxième repiquage, donne, dans les cas positifs, le champignon en culture pure. Sur huit hérissons autopsiés, nous en avons trouvé six parasités par des mycotorulées. Aucun animal ne présentait des signes de maladie attribuable aux champignons. Le parasitisme semblait donc indifférent pour ces animaux. Il résultait probablement d'une infection alimentaire, mais il est curieux de constater que les champignons se développent surtout dans la partie terminale du gros intestin, où on peut les 
trouver en très grand nombre. Notons que les mycotorulées résistent très bien à l'action des sucs digestifs, et peuvent même passer dans l'arbre respiratoire (cas 424 ).

Etude mycologique de ces souches. - Comme nous l'avons dit, l'isolement a été toujours fait sur Raulin acide en tube. Dans quelques cas, on obtient déjà un résultat positif au bout de 48 heures. Si la culture primaire n'est pas pure, cas le moins fréquent, il suffit de pratiquer un ou deux passages sur le même milieu pour arrêter le développement des bactéries. Les cultures sur Raulin peuvent être exclusivement constitućes par des blastospores. Les filaments peuvent n'apparaitre qu'au cours des premiers repiquages. Mais le véritable milieu réactif pour obtenir la filamentisation et par conséquent pour établir la diagnose du genre, est l'eau de pomme de terre, comme nous l'avons démontré (1).

L'etude morphologique complète des six souches provenant de hérissons, en milieu liquide et en milieu solide, suivant la méthode expliquée dans notre mémoire en collaboration avec le $\mathrm{D}^{\mathrm{r}}$ Langeron (2), nous a permis de constater qu'il s'agissait, dans cinq cas, du même champignon, et dans l'autre cas, d'une forme un peu différente au point de vue microscopique, mais identique au point de vue macroscopique. Ces derniers caractères macroscopiques des souches sont les suivants :

Cultures franchement crémeuses, humides, lisses, abondantes sur milieu gelosé glycosé à 2 p. 100. Toutes les souches poussent mieux à $37^{\circ}$ qu'à $25^{\circ}$, caractère commun à toutes les mycotorulées parasites de l'homme et des animaux. Le développement en profondeur est appréciable dans les tubes après quelques semaines.

Par l'aspect microscopique, les souches des hérissons 270, 801. 804, 808, 809 présentent la morphologie du genre Mycotorula. La souche du hérisson 424 appartient au genre Mycotoruloides.

Par l'ensemble de leurs caractères, les cinq souches de Mycotorula que nous avons isolées doivent rentrer dans l'espèce Mycotorula albicans (Ch. Robin 1853).

(1) Pour les détails concernant eette technique, nous remroyons le lecteur ì notre publication antérieure: Sur la filamentisation des Monilia. Ann, de parasilologie, VIII, 1930, p. 394-410.

(2) Langenon (M.) et Talice (I.-V.). - Nouvelles méthodes d'étude et essai de classification des champignons levuriformes. Ann. de parasitologie, X, 1932, p. $1-80$. 


\section{Rést mé}

Dans cette note, nous étudions le parasitisme du hérisson par les mycotorulées. A l'autopsie, nous avons constaté ce parasitisme six fois sur huit animaux. Le champignon, soit sous la forme blastosporée, soit sous la forme filamenteuse, a été trouvé tout le long du tube gastro-intestinal et une fois dans la trachée. Nous n'avons constaté, dans ces organes, ni lésions macroscopiques, ni lésions microscopiques. L'étude mycologique de ces 6 souches faite avec la technique que nous avons conseillée et qui a été adoptée aussi par Langeron et Talice, nous a conduit à identifier deux espèces : dans 5 cas le Mycotorula albicans (Ch. Robin 1853) et dans un cas Mycotoruloides sp.

\section{Bibliographite}

Blanchand (R.). - Maladies parasitaires, Paris, Masson, 1895.

Cataner (A.). - Présence d'un Monilia sur la langue de singes d'Algérie. $C$. $R$. Soc. biol., XCIII, 1925, p. 92-94.

Iangeron (M.) et Talice (R.-V.). - Nouvelles méthodes d'étude et essai de classification des champignons levuriformes. Ann. de parasit., $\mathrm{x}, 1932$, p. $1-80$.

Talice (R.-V.), - Sur la filamentisation des Monilia. Ann. de parasit., VIII, 1930, p. 394-410.

Thiry (G.). - Muguet spontané chez le singe. Langue pileuse brunc. Areh. parasil., XVI, 1913, p. 168-176.

"Seclion de mycologie du Laboraloire de parasilologie de la Facullé de médecine de Paris. 